\title{
Efetividade de jogo educativo para gestantes: conhecimento agregado e vivência das mulheres ${ }^{a}$
}

\author{
Effectiveness of an educational game for pregnant women: aggregated knowledge and the women's
} experience Eficacia del juego educativo para las mujeres embarazadas: conocimiento agregado y experiencia de
las mujeres

\author{
Carla Gisele D'Avila ${ }^{1}$ (c) \\ Kecia Larisse B. da Silva Oliveira² ${ }^{2}$ \\ Ronald Morais Castro ${ }^{2}$ (B) \\ Alfredo Almeida Pina-Oliveira ${ }^{1}$ (1) \\ Noélle de Oliveira Freitas ${ }^{1}$ (D) \\ Rosa Aurea Quintella Fernandes ${ }^{1}$ (D)
}

1. Universidade Guarulhos, Programa de Pós-Graduação em Enfermagem. Guarulhos, SP, Brasil.

2. Universidade Guarulhos, Curso de Graduação em Enfermagem. Guarulhos, SP, Brasil.

Autor correspondente:

Rosa Aurea Quintella Fernandes.

E-mail: fernands@uol.com.br

Recebido em 25/03/2021.

Aprovado em 11/08/2021.

DOI: https://doi.org/https://doi.org/10.1590/

2177-9465-EAN-2021-0078

\section{RESUMO}

Objetivos: verificar a efetividade do jogo educativo para gestantes sobre seus direitos durante o trabalho de parto e parto comparar o conhecimento das gestantes sobre seus direitos antes e após a aplicação do jogo e conhecer como foi para elas essa experiência. Método: estudo quantitativo com análise qualitativa secundária. A coleta de dados ocorreu entre julho $e$ setembro de 2019. Participaram 51 gestantes atendidas em duas Unidades Básicas de Saúde do município de Guarulhos. Utilizaram-se os testes Kolmogorov-Smirnov e o não-paramétrico Wilcoxon e nível de significância de 0,05. Adotou-se a análise lexical de conteúdo com apoio do software Interface de R pour les Analyses Multidimensionnelles de Textes et de Questionnaires. Resultados: a efetividade do jogo foi comprovada pela comparação da média do conhecimento das gestantes antes e após a atividade educativa $(Z=-5,924 ; p=0,000)$. Houve diferença significativa na comparação da avaliação do conhecimento das gestantes antes e após o jogo ( $\mathrm{p}=0,000)$. As respostas das gestantes enfatizaram a positividade da ação educativa e valorizaram as imagens do jogo como recurso educacional inovador. Conclusão e Implicações para a prática: O jogo mostrou-se efetivo para ser utilizado na orientação de gestantes de forma lúdica e demonstrou agregar conhecimento de modo imagético e participativo.

Palavras-chave: Educação em Saúde; Enfermagem; Jogos e Brinquedos; Parto; Saúde da Mulher.

\begin{abstract}
Objectives: to verify the effectiveness of an educational game for pregnant women about their rights during labor and delivery; to compare the knowledge of pregnant women about their rights before and after applying the game and to investigate how good this experience was for them. Method: quantitative study with secondary qualitative analysis. Data collection took place between July and September 2019. Participants were 51 pregnant women attended at two Primary Health Units of the city of Guarulhos. The Kolmogorov-Smirnov and the non-parametric Wilcoxon tests were used. The level of significance adopted was .05. Lexical content analysis was performed with the use of the Interface de R pour les Analyses Multidimensionnelles de Textes et de Questionnaires software. Results: the mean knowledge of the pregnant women after the educational activity was higher than the mean knowledge before the activity $(Z=-5.924 ; p=.000)$. There was a significant difference when comparing the assessment of the pregnant women's knowledge before and after the game $(p=.000)$. The responses of the pregnant women emphasized the positivity of the educational action and valued the images of the game as an innovative educational resource. Conclusion and Implications for practice: The study demonstrated that the game was effective for use in guiding pregnant women playfully and that knowledge was gained in an imagetic and participatory way.
\end{abstract}

Keywords: Health Education; Nursing; Games and Toys; Childbirth; Women's Health.

\section{RESUMEN}

Objetivos: verificar la eficacia del juego educativo para mujeres embarazadas sobre sus derechos durante el trabajo de parto y el parto; comparar el conocimiento de las mujeres embarazadas sobre sus derechos antes y después de aplicar el juego y saber cómo fue para ellas esta experiencia. Método: estudio cuantitativo con análisis cualitativo secundario. La recolección de datos se realizó entre julio y septiembre de 2019. Participaron 51 gestantes atendidas en dos Unidades de Atención Básicas de Salud de la ciudad de Guarulhos. Se utilizaron las pruebas de Kolmogorov-Smirnov y las pruebas no paramétricas de Wilcoxon. El nivel de significancia adoptado fue de 0,05. Se adoptó por el análisis de contenido léxico con el apoyo del Interface de $R$ pour les Analyses Multidimensionnelles de Textes et de Questionnaires. Resultados: el conocimiento medio de las embarazadas después de la actividad educativa fue mayor que el conocimiento medio antes de la actividad $(Z=-5,924 ; p=0,000)$. Hubo una diferencia significativa en la comparación de los conocimientos de las mujeres embarazadas antes y después del juego $(p=0,000)$. Las respuestas de las embarazadas destacaron la positividad de la acción educativa y valoraron las imágenes del juego como un recurso educativo innovador. Conclusión e implicaciones para la práctica: el juego demostró ser eficaz para ser utilizado en la orientación de las mujeres embarazadas de una manera lúdica y demostró añadir conocimiento de una manera imagética y participativa.

Palabras clave: Educación para la Salud; Enfermería; Juegos y Juguetes; Parto; Salud de la Mujer. 


\section{INTRODUÇÃO}

Nas últimas décadas, a assistência obstétrica sofreu grandes transformações, e novas práticas foram incorporadas baseadas em evidências científicas - as chamadas Boas Práticas, recomendadas pela Organização Mundial da Saúde (OMS). Assim, alguns procedimentos realizados anteriormente perderam força ou tiveram recomendação para serem abandonados, uma vez que as evidências científicas demonstraram sua ineficácia ou mesmo prejuízo às mulheres ${ }^{1,2}$.

Entretanto, apesar de decorridos alguns anos da implantação das Boas Práticas, muitos profissionais e instituições ainda as ignoram ou não as praticam. As dificuldades em consolidar modelos humanísticos e centrados na integralidade do cuidado nos serviços de saúde - associados à falta de informação e defesa de direitos humanos e das mulheres - contribuem para a persistência de um cenário em que gestantes que já tiveram outros filhos, desconheçam, muitas vezes, os novos procedimentos e ficam subjugadas à vontade dos profissionais no processo de parir ${ }^{3}$.

Nesse contexto, o pré-natal assume uma importância ímpar. A assistência pré-natal envolve mais do que as consultas, que são fundamentais para a saúde da mulher e da criança. Ele representa o lócus privilegiado para que a gestante participe ativamente de ações baseadas em informações fidedignas e atualizadas sobre o processo de parir, na discussão relativa aos seus direitos e no reconhecimento dos cuidados de seu filho ${ }^{1,4,5}$.

Uma das atividades que deve acontecer na assistência prénatal é o grupo de gestantes. Os grupos são recomendados como atividade educativa na assistência pré-natal, pois possibilitam que a gestante receba orientações dos profissionais de saúde, compartilhe conhecimentos prévios e experiências vivenciadas, e esclareça dúvidas que possam favorecer sua segurança e seu empoderamento no processo de nascer ${ }^{3,6,7}$.

O conhecimento que as gestantes precisam e podem adquirir em ações educativas, nos serviços de pré-natal, indicam a relevância da construção personalizada e colaborativa de um repertório individual de cuidado, capaz de sensibilizá-las a compreender seus direitos frente ao processo de nascimento e torná-las protagonistas de seu parto ${ }^{7}$. A mulher tem o direito sobre o seu corpo e, por isso, deve conhecer as práticas que Ihes são ou não recomendadas.

A prática educativa desenvolvida junto ao indivíduo favorece sua liberdade e traz benefícios que refletem em sua autonomia ${ }^{8}$. O compartilhar do saber e fazer - incluindo a educação em saúde - quebra tabus de autoritarismo entre profissionais e gestantes. Auxiliar as mulheres a adquirirem informações antes do processo de parturição é uma forma de humanização da assistência ${ }^{9}$.

A educação em saúde é uma estratégia valiosa que oportuniza e favorece o gestar e parir de forma prazerosa e segura, além de trazer maior tranquilidade à mulher. É uma abertura para educar que apoia a gestante, de modo a trazer tranquilidade para o período gestacional e um parto com menos intervenções ${ }^{4,9}$.

As tecnologias voltadas à educação em saúde são inúmeras. Nesse sentido, o emprego de jogos e brinquedos educativos na área de saúde representa um recurso tecnológico potente, que possibilita mudanças na melhoria da saúde, representando um instrumento para realizar a assistência integral $\left.\right|^{3,10}$.

D'Avila criou um jogo educativo para gestantes sobre seus direitos durante o trabalho de parto e parto, baseado nas boas práticas preconizadas pelo Ministério da Saúde (MS). O objetivo desse jogo educativo é preparar as mulheres para o processo de parir, de modo que elas compreendam claramente os procedimentos que podem ou não ser realizados, contribuindo para sua autonomia e protagonismo nesse processo. ${ }^{3}$

O diferencial desse jogo educativo, em relação aos poucos que existem envolvendo o trabalho de parto e parto, é a utilização de cartas com imagens. A maioria dos jogos disponível para esse público utiliza apenas frases sem imagem atrelada ${ }^{3}$.

O jogo educativo passou pelo processo de validação de conteúdo, realizada por juízes especialistas, sendo aplicado a número pequeno de gestantes, no escopo da validação ${ }^{3}$. Entretanto, não foi aplicado a uma amostra consistente de gestantes, nem avaliada sua eficácia em termos de agregar conhecimento às mulheres, o que motivou a realização deste estudo.

Assim, os objetivos do presente estudo foram: verificar a efetividade do jogo educativo para gestantes sobre seus direitos durante o trabalho de parto e parto; comparar o conhecimento das gestantes sobre seus direitos antes e após a aplicação do jogo; e conhecer como foi essa experiência para elas.

\section{MÉTODO}

Trata-se de estudo com abordagem quantitativa e utilizou análise secundária de dados qualitativos das respostas das gestantes participantes ${ }^{11}$. A pesquisa foi desenvolvida em duas Unidades Básicas de Saúde (UBS) do município de Guarulhos, estado de São Paulo, que atendem gestantes de risco habitual no pré-natal. Os dados foram coletados pela pesquisadora principal e por mais dois estudantes de graduação em Enfermagem, coautores do estudo, no período de julho a setembro de 2019.

A amostra foi por conveniência, e participaram as 51 gestantes que compareceram à consulta, no período determinado para a coleta dos dados, independentemente do trimestre gestacional. Para integrar a amostra, adotaram-se os seguintes critérios de inclusão: ter 18 anos ou mais, ser gestante de risco habitual e aceitar participar do jogo educativo. Não foram definidos critérios de exclusão.

$\mathrm{Na}$ coleta dos dados, foram utilizados dois instrumentos. O primeiro apresentava dados sociodemográficos e obstétricos: idade, raça/etnia autodefinida, situação conjugal, escolaridade, renda familiar, atividade profissional, religião, gestação, número de filhos, número de consultas pré-natal, participação em grupo de gestantes, e orientações sobre o trabalho de parto e parto. O segundo foi um questionário, criado para este estudo, com 17 questões fechadas que valiam um ponto por acerto e envolviam o conteúdo das cartas do jogo. Havia duas questões abertas: uma sobre como foi para elas (gestantes) participarem do jogo educativo e outra relativa à evocação de uma a cinco palavras que melhor expressassem a experiência de participar do jogo, que constituíram a coleta dos dados qualitativos do estudo. Essas duas questões foram respondidas após a participação das gestantes no jogo educativo. 
O jogo educativo utilizado no estudo foi desenvolvido com o objetivo de preparar as mulheres para o processo de parir, a fim de que elas compreendam os procedimentos a que serão submetidas $^{3}$. O jogo consiste em 44 cartas, sendo 22 com frases afirmativas sobre as boas práticas na assistência ao parto e os direitos das mulheres nos períodos de pré-parto, parto e pósparto, e 22 imagens correspondentes a cada afirmativa. Tratase de um jogo de associação; sendo assim, as participantes devem correlacionar a afirmativa de uma carta com a imagem correspondente. $\mathrm{O}$ facilitador do jogo deve estimular a discussão do conteúdo das cartas, com o objetivo de dirimir dúvidas e adequar as informações em prol de fortalecer boas práticas ${ }^{3}$.

A coleta dos dados foi realizada em três etapas consecutivas: antes, durante e após a participação das gestantes no jogo. As mulheres foram abordadas na sala de espera enquanto aguardavam a consulta pré-natal, sendo então convidadas a participar da pesquisa. O processo envolvia responder às questões do questionário, participar do jogo em grupos de mulheres, e responder novamente ao questionário acrescido das perguntas abertas para a análise de dados qualitativos.

A coleta dos dados durou aproximadamente 50 minutos para cada participante, incluindo o jogo em grupo. Não houve recusa de nenhuma gestante em participar, e todas ficaram muito interessadas, pois nas duas UBS selecionadas não havia grupo educativo para gestantes.

Os dados quantitativos foram digitados no programa Microsoft Excel $\circledR$ versão 2016, e analisados no programa Statistical Package for the SocialSciences (SPSS)® versão 20 para Windows $\unrhd$. Quanto aos dados sociodemográficos, obstétricos e das respostas quantitativas de avaliação do conhecimento das mulheres, foi realizada análise descritiva. A normalidade dos dados foi avaliada por meio do teste de Kolmogorov-Smirnov. Como não se identificou distribuição normal dos dados $(p<0,05)$, foi utilizado o teste nãoparamétrico Wilcoxon para comparar os resultados do conhecimento das gestantes antes e após a aplicação do jogo educativo. Testou-se a hipótese de que as gestantes apresentariam maior conhecimento após a aplicação do jogo educativo. O nível de significância adotado foi de 0,05.

Considerando a natureza dos dados qualitativos obtidos, optouse pela análise lexical do conteúdo das respostas das 51 gestantes, a fim de identificar significados e tendências sobre a experiência de participar do jogo educativo por meio de estatísticas textuais. Destarte, entende-se que essa análise secundária qualitativa complementou os resultados quantitativos e, para minimizar a ausência de um referencial teórico específico, recorreu-se à discussão baseada em estudos científicos atuais que utilizaram jogos e educação em saúde.

Ressalta-se que as respostas às questões abertas do estudo foram transcritas de modo fidedigno à fala das gestantes, pelos dois estudantes pesquisadores, e sob supervisão de um pesquisador com produção em pesquisas qualitativas na interface entre Enfermagem, Promoção da Saúde e Educação.

Os dados coletados nessa etapa foram armazenados e organizados por meio de planilha em Microsoft Excel@e transformados em documento TXT no Microsoft Bloco de Notas $₫$, versão 1903, e com codificação Unicode Transformation Format 8-bit (UTF-8). Após esse tratamento dos dados, utilizou-se o software Interface de Rpour les Analyses Multidimensionnelles de Textes et de Questionnaires (IRAMUTEQ), em sua versão 0.7 alpha 2 , a fim de extrair estatísticas textuais para a elaboração de gráficos comparativos de substantivos, adjetivos, verbos mais representativos do objeto estudado, conforme recomendações de Laurence Bardin sobre a utilização de palavras plenas na análise lexical e sintática do conteúdo de um corpus textual ${ }^{12}$.

Para as análises no IRAMUTEQ, elaboraram-se linhas de comando das respostas das gestantes com as seguintes variáveis: gestante, idade, situação conjugal, escolaridade, remuneração, religião, gestação e participação prévia em grupo de gestantes. Selecionaram-se o Dendrograma da Análise de Similitude, com a delimitação por comunidades (cores diferentes), e a Matriz Prototípica da avaliação da Ordem de Evocação Média (OEM) de até cinco palavras relacionadas à participação delas no jogo, as quais foram anotadas na sequência exata que as mulheres mencionaram à dupla de estudantes pesquisadores.

A pesquisa foi autorizada pela Secretaria de Saúde do município de Guarulhos, Estado de São Paulo, e aprovada pelo Comitê de Ética em Pesquisa (CEP) sob Parecer n.ㅇ․ 3.362.305,

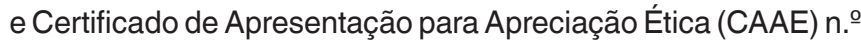
10492019.0.0000.5506. Todas as mulheres assinaram o Termo de Consentimento Livre e Esclarecido (TCLE).

\section{RESULTADOS}

A amostra foi constituída por 51 gestantes. O perfil das participantes pode ser assim delineado: média de idade 27,1 anos, mínimo de 18 e máximo de 45; 45\% estavam no $3^{\circ}$ trimestre de gestação; renda familiar média de $\mathrm{R} \$ 1.672,00$. Mais da metade definiu-se parda (57\%), tinha companheiro (88,2\%), possuía ensino médico completo $(76,4 \%)$, não exercia atividade remunerada $(62,8 \%)$, era evangélica (56,9\%) e multigesta $(56,9 \%)$.

A maioria $(86,2 \%)$ referiu não participar de grupo de gestante e não receber orientações sobre o trabalho de parto $(82,3 \%)$, e sobre o parto $(86,2 \%)$.

Nas respostas às questões formuladas, com base nas cartas do jogo, o percentual de acerto das gestantes, antes da ação educativa (jogo), foi baixo; apenas 6 questões (35,2\%) tiveram acerto $\geq a \quad 80 \%$. As questões mais acertadas foram: a mulher deve ser informada sobre tudo que vai acontecer com ela durante o trabalho de parto e parto; e, durante o trabalho de parto, parto e pós-parto, a mulher tem direito de ter acompanhante com $98 \%$ de acertos.

Outras questões com boa assertiva foram sobre os procedimentos e cuidados pessoais: na maternidade, o profissional pode realizar o exame de toque vaginal $(90,2 \%)$; a gestante, durante o trabalho de parto, pode tomar banho $(80,4 \%)$. As questões referentes aos direitos do bebê mais acertados foram: após o nascimento, o bebê deve ficar junto da mãe no quarto o tempo todo $(88,2 \%)$; e, depois que o bebê nascer, ele deve mamar na mãe durante a primeira hora de vida $(80,4 \%)$.

Os resultados após a ação educativa (jogo) se inverteram, uma vez que o percentual de acerto foi superior $\geq$ a $90 \%$ em $94,1 \%$ das questões. 


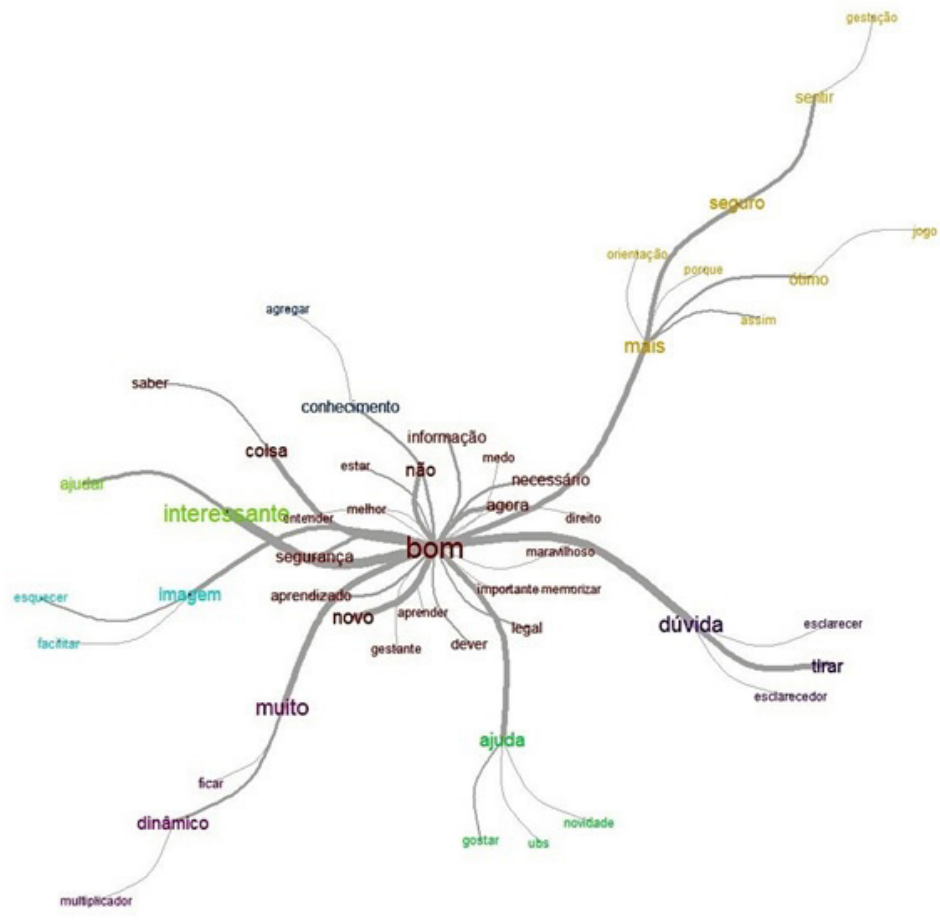

Figura 1. Dendrograma com comunidades relacionado a experiências das gestantes no "Jogo educativo para gestantes". Guarulhos, 2019.

Fonte: Próprios autores com base na análise de similitude do software IRAMUTEQ.

Tabela 1. Comparação da avaliação do conhecimento antes e depois da atividade educativa (n=51). Guarulhos, São Paulo, Brasil 2019.

\begin{tabular}{cccccc}
\hline Variável & $\mathrm{N}$ & Média & D.P. $^{\text {a }}$ & Mediana & p-valor $^{\text {b }}$ \\
\hline Conhecimento antes do jogo & 51 & 29,8 & 2,2 & 30 & 0,000 \\
Após o jogo & 51 & 33,3 & 1,3 & 34 & \\
\hline
\end{tabular}

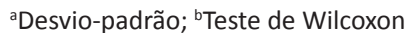

Fonte: Elaborada pelos autores

Tabela 2. Avaliação da satisfação com o jogo e novas informações recebidas ( $n=51)$. Guarulhos, SP, Brasil, 2019.

\begin{tabular}{|c|c|c|}
\hline Variáveis & $\mathrm{N}$ & $\%$ \\
\hline \multicolumn{3}{|c|}{ Gostaram de participar do jogo } \\
\hline Muito & 40 & 78,4 \\
\hline Mais ou Menos & 09 & 17,7 \\
\hline Pouco & 02 & 3,9 \\
\hline \multicolumn{3}{|c|}{ Recebeu novas Informações } \\
\hline Muitas & 44 & 86,2 \\
\hline Regular & 04 & 7,9 \\
\hline Poucas & 03 & 5,9 \\
\hline
\end{tabular}

Fonte: Elaborada pelos autores
Observa-se, na Tabela 1, que o teste de Wilcoxon identificou a média do conhecimento das gestantes após a atividade educativa maior do que a média do conhecimento antes da atividade $(Z=$ $-5,924 ; p=0,000)$. Houve diferença significativa na comparação da avaliação do conhecimento das gestantes antes e após o jogo ( $p=0,000)$, confirmando a hipótese de que o conhecimento delas seria maior após a ação educativa.

A Tabela 2 evidencia a avaliação das gestantes na participação do jogo. A maioria $(78,4 \%)$ gostou muito de participar e avaliou que recebeu muitas informações novas $(86,2 \%)$.

De modo complementar à Tabela 2 , a análise lexical evidenciou estatísticas textuais para compreender a experiência das gestantes. O resumo do IRAMUTEQ indicou 51 textos, com 921 ocorrências, 169 formas e 70 hápax (palavras de ocorrência única), com a média geral de ocorrências por texto de 18,06.

A análise de similitude (Figura 1) permitiu visualizar as coocorrências das palavras entre si e agrupamentos de termos 
que favorecessem o entendimento sobre potenciais relações e aspectos que se circunscrevem à experiência de jogar para promover a saúde das gestantes.

A palavra central "bom" aglomerou-se com termos que primam pela aprendizagem das gestantes, segurança nas informações e modos de entender melhor os conteúdos relevantes para o seu cuidado sobre o trabalho de parto e parto.

No tocante à ação educativa em saúde, as gestantes referiram a segurança nas orientações, o manejo das emoções na gravidez, a dinamicidade do jogo educativo, as imagens favoráveis à memorização, a abertura para o esclarecimento de dúvidas, a construção de conhecimentos agregadores, a inovação nos modos de cuidar e a necessidade de incorporar estratégias similares nas UBS.

A análise de similitude destaca a relevância dos aspectos imagéticos e da utilização de abordagens dialógicas para engajar os participantes, por meio de materiais educacionais que inovam o ensino e a aprendizagem em contextos educacionais, com foco no incremento de conhecimentos sobre boas práticas e maneiras de defender os direitos das gestantes.

A matriz prototípica representa a frequência $(f)$ e a ordem de evocação média (OEM), e demonstra a relevância específica de cada palavra associada pelas gestantes após sua participação no jogo educativo (Tabela 3 ). Nesse sentido, evidenciou-se reação positiva com relação ao jogo educativo e o uso de uma estratégia inédita para avaliação de reação inspirada na Técnica de Associação Livre de Palavras (TALP) ${ }^{13}$.
No núcleo central, os termos foram mais frequentes $(<=$ $6,96)$ e prontamente mencionados pelas gestantes $(<=2,35)$. Os termos da primeira periferia foram frequentes, mas relatados em posições acima de 2,35 na OEM, e indicaram o quanto o jogo representa uma abordagem inovadora, dinâmica e necessária para promover aprendizagem significativa. Na zona de contraste, os termos foram menos frequentes, mas rapidamente evocados para expressar a construção de conhecimentos e prestatividade dos profissionais que realizaram o jogo educativo. Por fim, na segunda periferia encontram-se os termos menos frequentes e posicionados acima de 2,35 na OEM, para reforçar a avaliação positiva e a sugestão, visando maior incorporação do jogo educativo no cuidado em saúde das gestantes.

\section{DISCUSSÃO}

A importância dos grupos de gestantes no pré-natal está definitivamente comprovada em publicações ${ }^{14-16}$. A discussão de temas ligados ao parto e aos cuidados do recém-nascido enriquecem e empoderam as mulheres, tornando-as mais seguras e preparadas para o processo de parir ${ }^{14-16}$. O grupo de gestantes é uma tecnologia leve, costuma ter baixo custo, possibilita ganhos importantes para a saúde da mulher, complementa as informações da consulta e eleva a qualidade da assistência. Dessarte, essa abordagem grupal torna-se um espaço privilegiado para discutir medos, expressar inseguranças, esclarecer dúvidas, compartilhar

Tabela 3. Matriz da análise prototípica da avaliação de reação referente à participação no "Jogo educativo para gestantes". Guarulhos, 2019.

\begin{tabular}{|c|c|c|c|c|c|}
\hline \multicolumn{3}{|c|}{ Núcleo Central } & \multicolumn{3}{|c|}{ Primeira Periferia } \\
\hline Bom & 30 & 1,5 & Ajuda & 16 & 2,4 \\
\hline Interessante & 19 & 2,1 & Novo & 13 & 2,7 \\
\hline Ótimo & 11 & 1,5 & Necessário & 10 & 3,4 \\
\hline Segurança & 10 & 2,3 & Aprendizado & 8 & 3,1 \\
\hline \multicolumn{3}{|c|}{ Zona de contraste } & \multicolumn{3}{|c|}{ Segunda Periferia } \\
\hline Palavra & $f(<6,96)$ & $\operatorname{OEM}(>2,35)$ & Palavra & $f(<6,96)$ & $\operatorname{OEM}(>6,96)$ \\
\hline Conhecimento & 3 & 2,3 & Legal & 5 & 3,8 \\
\hline Agregador & 2 & 1,5 & Dinâmico & 3 & 3,3 \\
\hline- & - & - & Multiplicadora & 3 & 3 \\
\hline- & - & - & Eficiente & 2 & 2.5 \\
\hline- & - & - & Produtivo & 2 & 2.5 \\
\hline- & - & - & Feliz & 2 & 3 \\
\hline
\end{tabular}

Fonte: Elaborada pelos autores. 
experiências e refletir sobre dificuldades relacionados, em especial, a gestantes adolescentes e primíparas ${ }^{17}$.

Entretanto, os resultados desta pesquisa demonstram a não valorização dessa estratégia, uma vez que a maioria das gestantes respondeu não participar de grupos, e mais de $80 \%$ delas informaram não ter recebido orientações sobre o trabalho de parto e parto, visto que a atividade em grupo para gestante não existe nas Unidades pesquisadas, fato que certamente repercutiu nos resultados pífios identificados na etapa anterior ao jogo educativo.

Todavia, perde-se a oportunidade de melhorar o conhecimento das mulheres sobre seus direitos, as boas práticas na assistência ao trabalho de parto e parto e os cuidados com o filho, o que Ihes daria maior segurança no enfrentamento das situações no momento da internação.

O jogo proposto mostrou-se eficaz, uma vez que houve melhora estatisticamente significativa no conhecimento das mulheres antes e após a sua aplicação. A maioria das gestantes gostou de participar da atividade e reconheceu ter apreendido novos conhecimentos. Desse modo, o jogo cumpriu a ideia de trabalhar em direção a um objetivo coletivo, e a ação educativa caracterizou-se como estratégia pedagógica na área da saúde ${ }^{18}$.

Adotou-se a concepção de educação em saúde como uma prática social que prima pelo diálogo, pela problematização do contexto real, pelo respeito às pessoas e pela troca de saberes, sobretudo durante o pré-natal ${ }^{19}$. Nesse sentido, o presente jogo representa um recurso educacional que permitiu a alternância entre uma ação informativa para promoção do conhecimento das gestantes partícipes, e uma ponte possível para uma ação educativa mais complexa e com potencial de transformação individual e coletiva no âmbito da atenção primária em saúde.

A efetividade do jogo, como ação educativa na incorporação de conhecimento, foi também comprovada em estudo semelhante ao atual, que a verificou no conhecimento de adolescentes sobre contracepção ${ }^{20}$. Outro estudo com adolescentes concluiu que o jogo foi uma tecnologia educativa que agregou saberes para mães adolescentes nos cuidados com o filho ${ }^{21}$.

Publicações evidenciam que o jogo educativo contribui para a programação de contingências de ensino eficazes e eficientes, tanto na educação em saúde de pacientes quanto de profissionais ${ }^{22,23}$. Após a validação de conteúdo de jogos, alguns autores concluíram que esse tipo de recurso educacional na área de saúde provoca mudanças de atitude e comportamento naqueles que a utilizam em diferentes áreas da saúde ${ }^{24,25}$.

No tocante aos aspectos qualitativos, as literaturas nacional e internacional apresentam uma lacuna sobre avaliações das experiências em jogos educativos relacionados ao cuidado de gestantes.

Portanto, o presente estudo indica uma área promissora para novas e aprofundadas investigações sobre a usabilidade dessa tecnologia educacional, e a experiência vivenciada por usuários de diferentes serviços de saúde, assim como amplia o uso das funcionalidades do IRAMUTEQ com a escolha da Matriz Prototípica, conforme recomendações de pesquisadores da área ${ }^{26}$.

Em um estudo realizado em Bangladesh, os pesquisadores identificaram temas relevantes para as participantes sobre a adoção de grupos de cuidado no pré-natal, sendo que a abordagem compreensiva dos profissionais de saúde, a socialização, o aprender a lidar com desconfortos, a relação interpessoal nos serviços, o preparo para o parto e a presença participativa de mães compreendem aspectos centrais para ações educativas em saúde, como a proposta do jogar no presente estudo ${ }^{27}$.

A percepção de gestantes e puérperas no contexto da atuação de odontopediatras, na Estratégia Saúde da Família, enfatiza a relevância de ações educativas para promover a saúde das mulheres e crianças, sem desconsiderar suas famílias e comunidades ${ }^{28}$. Por aproximação teórica, o uso de jogos que exploram elementos imagéticos, colaboração e autonomia das participantes pode fortalecer estratégias educativas na Atenção Primária à Saúde.

\section{CONCLUSÃO E IMPLICAÇÕES PARA A PRÁTICA}

Pelos resultados obtidos, conclui-se que o jogo foi efetivo e promoveu uma experiência positiva e motivadora para as mulheres que participaram dessa ação educativa, no contexto da Atenção Primária à Saúde.

Assim, espera-se que a utilização desse jogo, nas orientações do pré-natal, contribua para a autonomia da mulher e a liberte das decisões unilaterais que ocorrem nos procedimentos durante o trabalho de parto e parto, na maioria das vezes, por falta de conhecimento sobre seus direitos.

Neste estudo, a coleta de dados qualitativos por meio de inquérito foi entendida como uma limitação e pode ter induzido respostas automáticas ou "esperadas" das gestantes. Por essa razão, entende-se que a análise lexical consiste em uma modalidade coerente para explorar o conteúdo das respostas obtidas com as 51 gestantes partícipes.

Considerou-se o tamanho amostral outra limitação do estudo, assim como a coleta de dados sobre o conhecimento agregado ter ocorrido imediatamente após o jogo. Assim, sugere-se que o jogo seja empregado em outras pesquisas e com maior espaçamento de tempo entre a ação educativa e o teste.

Empregar recursos educacionais como esse jogo de cartas com imagens representativas do período gravídico pode tornar as orientações em grupos de gestantes mais efetivas, engajadoras e visualmente atrativas, com foco na participação ativa das mulheres em cenários que cuidam, protegem e educam nas Redes de Atenção à Saúde.

\section{CONTRIBUIÇÕES DOS AUTORES}

Desenho do estudo. Carla Gisele D’Avila. Kécia Larisse Bezerra da Silva Oliveira. Ronald Morais Castro. Rosa Aurea Quintella Fernandes.

Coleta ou produção dos dados. Carla Gisele D’Avila. Kecia Larisse Bezerra da Silva Oliveira. Ronald Morais Castro.

Análise de dados. Kecia Larisse Bezerra da Silva Oliveira. Ronald Morais Castro. Alfredo Almeida Pina-Oliveira. Noélle de Oliveira Freitas. Rosa Aurea Quintella Fernandes. 
Interpretação dos resultados. Kecia Larisse Bezerra da Silva Oliveira. Ronald Morais Castro. Alfredo Almeida Pina-Oliveira. Noélle de Oliveira Freitas. Rosa Aurea Quintella Fernandes.

Redação e revisão crítica do manuscrito. Carla Gisele D’Avila. Kecia Larisse Bezerra da Silva Oliveira. Ronald Morais Castro. Alfredo Almeida Pina-Oliveira. Noélle de Oliveira Freitas. Rosa Aurea Quintella Fernandes.

Aprovação da versão final do artigo. Carla Gisele D’Avila. Kecia Larisse Bezerra da Silva Oliveira. Ronald Morais Castro. Alfredo Almeida Pina-Oliveira. Noélle de Oliveira Freitas. Rosa Aurea Quintella Fernandes.

Responsabilidade por todos os aspectos do conteúdo e a integridade do artigo publicado. Carla Gisele D’Avila. Kecia Larisse Bezerra da Silva Oliveira. Ronald Morais Castro. Alfredo Almeida Pina-Oliveira. Noélle de Oliveira Freitas. Rosa Aurea Quintella Fernandes.

\section{EDITOR ASSOCIADO}

\author{
Candida Primo Caniçali (D)
}

\section{EDITOR CIENTÍFICO}

\author{
Ivone Evangelista Cabral (D)
}

\section{REFERÊNCIAS}

1. World Health Organization. WHO recommendations: intrapartum care for a positive childbirth experience. Geneva:WHO; 2018 [citado 2021 mar 20]. Disponível em: https://apps.who.int/iris/handle/10665/260178

2. Leal MC, Pereira APE, Theme Fa MMT, Dias MAB, Nakamura-Pereira $\mathrm{M}$, Bastos $\mathrm{MH}$ et al. Obstetric interventions during labor and childbirth in Brazilian low-risk women. Cad Saude Publica. 2014;30(1, suppl 1):1747. http://dx.doi.org/10.1590/0102-311X00151513. PMid:25167177.

3. D'Avila CG, Puggina AC, Fernandes RAQ. Construction and validation of an educational game for pregnant women. Esc Anna Nery. 2018;22(3):e20170300. http://dx.doi.org/10.1590/2177-9465-ean-2017-0300.

4. Duarte SJH, Almeida EP. O papel do enfermeiro do programa saúde da família no atendimento pré-natal. R Enferm Cent O Min. 2014;4(1):1029-35.

5. Guedes GW, Sousa MNA, Lima TNFA, Lima MNFA, Davim RMB, Costa TS. Pregnant women knowledge about the benefits of normal birth in prenatal consultation. Rev Enferm UFPE online. 2016;10(10):3860-7.

6. Matos GC, Demori CC, Escobal APL, Soares MC, Meincke SMK, Gonçalves KD. Groups of pregnant women: space for a humanization of labor and birth. Rev Fund Care Online. 2017 abr/jun;9(2):393-400.

7. Souza EVA, BasslerTC, Taveira AG. Health education in the empowerment of the pregnant woman. Rev enferm UFPE online. 2019;13(5):1527-31.

8. Braga CJM, Pantoja LDM, Bachur TPR, Aragão GF. Jogo de cartas como estratégia para o ensino de doenças autoimunes na graduação médica. Rev Electron Comun Inf Inov Saude. 2019;13(3):594-607. http://dx.doi.org/10.29397/reciis.v13i3.1483.

9. Alves FLC, Castro EM, Souza FKR, Lira MCPS, Rodrigues FLS, Pereira LP. Group of high-risk pregnant women as a health education strategy. Rev Gaúcha Enferm. 2019;40:e20180023. http://dx.doi.org/10.1590/19831447.2019.20180023.

10. Ferreira MA, Fernandes MC, Leandro GB, Rolim ALG, Andrade ME. Educational technologies in adolescent empowerment about depression. Rev enferm UFPE online. 2019;13(1):275-80. http://dx.doi. org/10.5205/1981-8963-v13i1a237881p275-280-2019.

11. Heaton J. Secondary analysis of qualitative data: an overview. Hist Soc Res. 2008;33(3):33-45.

12. Bardin L. Análise de conteúdo. Lisboa: Edições 70; 2016. 229 p.

13. Coutinho MPL, Bú E. A Técnica de Associação Livre de Palavras sobre o prisma do software Tri-Deux-Mots (version 5.2). Rev Campo do Saber. 2017;3(1):219-43

14. Frigo LF, Silva RM, Boeira GS, Manfio F. A importância dos grupos de gestante na atenção primária: um relato de experiência. Rev Epidemio Control Infect. 2012;2(3):113-4. http://dx.doi.org/10.17058/reci.v2i3.2745.

15. Domingues F, Pinto FS, Pereira VM. Grupo de gestantes na atenção básica: espaço para construção do conhecimento e experiências na gestação. Rev Fac Ciênc Méd Sorocaba. 2018;20(3):150-4. http:// dx.doi.org/10.23925/1984-4840.2018v20i3a6.

16. Henriques AHB, Lima GMB, Trigueiro JVS, Saraiva AM, Pontes MGA Cavalcanti JRD. Grupo de gestantes: contribuições e potencialidades na complementaridade da assistência pré-natal. Rev Bras Promoç Saúde. 2015;28(1):23-31. http://dx.doi.org/10.5020/18061230.2015. p23.

17. Queiroz MVO, Menezes GMD, Silva TJP, Brasil EGM, Silva RM. Pregnant teenagers' group: contributions to prenatal care. Rev Gaúcha Enferm. 2016;37:e2016-0029.

18. Castillo Lizardo JM, Rodríguez-Morán M, Guerrero-Romero F. El juego como alternativa para La enseñanza de conceptos básicos de salud Rev Panam Salud Publica. 2001;9(5):311-4. http://dx.doi.org/10.1590/ S1020-49892001000500005. PMid:11476020.

19. Costa APC, Bustorff LACV, Cunha ARR, Araújo VS. Contribuição do pré-natal para o parto vaginal: percepção de puérperas. Rev Rene. 2011;12(3):548-54.

20. Praxedes MLS, Queiroz MVO, Vieira RP. Efetividade de jogo educativo sobre contracepção com adolescentes escolares: estudo quaseexperimental. J Nurs. 2019;18(4).

21. Andrade RD, Hilário JSMI, Santos JS, Silva JP, Fonseca LMM, Mello DF Maternal-child nursing care for adolescent mothers: health education. Rev Bras Enferm. 2020;73(4):e20180769. http://dx.doi.org/10.1590/00347167-2018-0769. PMid:32520091.

22. Panosso MG, Souza SR, Haydu VB. Características atribuídas a jogos educativos: uma interpretação Analítico-Comportamental. Rev Assoc Bras Psic Esc Educ. 2015;19(2):2015:233-41.

23. Dias JD, Mekaro MS, Lu JKC, Otsuka JL, Fonseca LMM, ZemMascarenhas SH. Serious game development as a strategy for health promotion and tackling childhood obesity. Rev Latino-Am Enfermagem. 2016;24:e2759. http://dx.doi.org/10.1590/1518-8345.1015.2759.

24. Daniel ACQG, Veiga EV, Machado JP, Mafra ACCN, Cloutier L. Effect of an educational program for the knowledge and quality of blood pressure recording. Rev Lat Am Enfermagem. 2019;27:e3179. http:// dx.doi.org/10.1590/1518-8345.3011.3179. PMid:31596414.

25. Mariano MR, Rebouças CBA, Pagliuca LMF. Educative game on drugs for blind individuals: development and assesment. Rev Esc Enferm USP. 2013;47(4):930-6. http://dx.doi.org/10.1590/S0080-623420130000400022. PMid:24310692.

26. Souza MAR, Wall ML, Thuler ACMC, Lowen IMV, Peres AM. The use of IRAMUTEQ software for data analysis in qualitative research. Rev Esc Enferm USP. 2018;52:e03353. PMid:30304198.

27. Sultana M, Ali N, Akram R, Jahir T, Mahumud RA, Sarker AR et al. Group prenatal care experiences among pregnant women in a Bangladesh community. PLoS One. 2019;14(6):e0218169. http://dx.doi.org/10.1371/ journal.pone.0218169. PMid:31188891.

28. Herval ÁM, Oliveira FPSL, Machado KM, Vasconcelos M, Ferreira RC, Ferreira EF et al. Mothers' perception about health education in brazilian primary health care: a qualitative study. Int J Paediatr Dent. 2019;29(5):669-76. http://dx.doi.org/10.1111/ipd.12489. PMid:30817034

\footnotetext{
a Extracted from the Course Completion work, "Effectiveness of an educational game for pregnant women: rights in labor and delivery", presented in the Undergraduate Nursing Course of Guarulhos University, in 2019. Authored by Kecia Larissa Bezerra da Silva Oliveira and Ronald Morais Castro, under the guidance of Prof. Dr Rosa Aurea Quintella Fernandes.
} 